\title{
Investigating the Unity and Disunity of Scientific Explanation
}

\author{
Erik Weber ${ }^{1} \cdot$ Henk W. de Regt ${ }^{2} \cdot$ Dingmar van Eck $^{1,3}$ \\ Published online: 21 September 2020 \\ (c) Springer Nature B.V. 2020
}

\section{Plurality of Structures in Scientific Explanation}

The starting point for this special issue is that explanations as they occur in scientific practice exhibit a plurality of structures. The explananda are at different levels (e.g. events, regularities, laws) and of different types (e.g. plain facts, contrasts, similarities). Likewise, the explanantia can have different ingredients (e.g. causes, constraints, laws, mechanisms) and different types of organisation (e.g. deductive and/or inductive arguments, causal models).

We give a brief illustration of this plurality. We start with an example that Paul Humphreys uses in his book The Chances of Explanation (1989). Suppose that Albert has died and we want an explanation for this event. An explanation could be:

Albert's death occurred because of his infection with the plague bacillus, despite the administration of tetracycline to him. (p. 100).

In this explanation, the explanans contains a positive causal factor for the explanandum (the infection) and a negative causal factor (the administration of tetracycline). Let us contrast this with an example about thermal expansion. The expansion of aluminium rods is governed by the law $d \mathrm{~L}=0.0000222 \times \mathrm{L}_{0} \mathrm{x} d \mathrm{t}$, where $d \mathrm{~L}$ is the expansion (in metre), $\mathrm{L}_{0}$ the initial length (in metre) of the rod and $d$ t the temperature difference (in ${ }^{\circ} \mathrm{C}$ ). 0.0000222 is the coefficient of linear thermal expansion of aluminium. With this background knowledge we can construct the following example:

$\mathrm{C}_{1}$ : This aluminium rod was heated from $50{ }^{\circ} \mathrm{C}$ to $250{ }^{\circ} \mathrm{C}$.

$\mathrm{C}_{2}$ : This aluminium rod has an initial length (at $50^{\circ} \mathrm{C}$ ) of $1 \mathrm{~m}$.

L: For all aluminium rods: if they are heated from $50{ }^{\circ} \mathrm{C}$ to $250{ }^{\circ} \mathrm{C}$ and their initial length is $1 \mathrm{~m}$, then they are $4,44 \mathrm{~mm}$ longer at $250{ }^{\circ} \mathrm{C}$.

Erik Weber

Erik.Weber@ugent.be

Henk W. de Regt

henk.deregt@ru.nl

Dingmar van Eck

d.vaneck@uva.nl; Dingmar.vanEck@ugent.be

1 Centre for Logic and Philosophy of Science, Ghent University, Ghent, Belgium

2 Institute for Science in Society, Radboud University, Nijmegen, The Netherlands

3 Institute for Logic, Language \& Computation, University of Amsterdam, Amsterdam, The Netherlands 
$\mathrm{E}$ : This aluminium rod is $4,44 \mathrm{~mm}$ longer than it was before it was heated.

This explanation also mentions two causes (temperature increase and initial length) but without dividing them into positive (contributing) and negative (counteracting) ones, because that distinction makes no sense here. Two other differences with the first example are that there is a covering law used here, and that the explanation has the form of an argument. In the first example there is no covering law, and the explanation does not have the form of an argument. These two examples thus illustrate that scientific explanations exhibit a plurality of structures. There are many types of explanation, with different ingredients and organisation of the explanans, that address the same type of explanandum (e.g. a plain particular fact as in the two examples.

\section{Explanatory Pluralism}

Most philosophers of explanation nowadays are explanatory pluralists. Explanatory pluralism is the view that adequate scientific explanations can have many different structures. According to an explanatory pluralist there is no 'canonical form' in which all legitimate scientific explanations can be represented without losing crucial information. Note that we have used evaluative terms ('adequate', 'legitimate') in this characterisation of explanatory pluralism. We do so on purpose because explanatory pluralism entails the normative endorsement of the plurality of types of explanation in science. An explanatory monist can acknowledge that scientists produce different types of explanation, but consider only one type as really adequate. For instance, an explanatory monist of the derivationist kind may insist that only the second explanation above is legitimate, while the first is not adequate because there is no covering law and no argument.

An explanatory pluralist can explore a number of philosophical research routes. A first option is to use philosophical models of explanation in order to describe and evaluate the explanatory practices in a specific scientific domain. This approach is compatible with explanatory pluralism because of the focus on one or a few scientific disciplines. There are no general claims about the structure of all scientific explanations. As we will see below in Sect. 3, this is the approach that is taken in two of the papers in this volume.

A second option is to aim at generic insights on scientific explanation without falling back into explanatory monism. Let us clarify how this is possible. Suppose you adopt the view that all (legitimate) scientific explanations relating to particular facts are 'causation-based', in the sense that the following two conditions hold:

(1) the explanation presupposes the existence of causal relations; ontologically speaking it is grounded in causal relations.

(2) the explanation gives some information about the causes of the explanandum, and this information is crucial to make the explanation work.

This could be a general insight on explanations of particular facts, and is compatible with explanatory pluralism because there are many types of causal explanations (for instance, the examples in Sect. 1 belong to a different type). As we will clarify below in Sect. 4, the three other articles in this issue aim at providing such generic insights. 


\section{Explanation in Mathematics and Cognitive Science}

In 'Unificatory Understanding and Explanatory Proofs' Joachim Frans engages in the debate on the distinction between explanatory proofs and non-explanatory proofs in mathematics. He defends four claims: (1) understanding is a condition for explanation, (2) unificatory understanding is a type of explanatory understanding, (3) unificatory understanding is valuable in mathematics, and (4) mathematical proofs can contribute to unificatory understanding. As a result, in a context where the epistemic aim is to unify mathematical results, it is fruitful to make a distinction between proofs based on their explanatory value.

In 'The Multiplicity of Explanation in Cognitive Science' Raoul Gervais argues that explaining cognitive behaviour can be achieved through 'hybrid explanatory inferences': inferences that posit mechanisms, but also draw on observed regularities. Moreover, these inferences can be used to achieve unification. Hence, in the cognitive sciences, explanatory pluralism represents a way to achieve unification.

\section{Generic Insights on Scientific Explanation}

In 'Model Explanation vs. Model-Induced Explanation', Insa Lawler and Emily Sullivan start from the observation that many (but not all!) scientific explanations are modelbased. But what are these 'model-based explanations'? Are they special, for example, because they challenge the factivity of explanation? Lawler and Sullivan study three paradigmatic cases of alleged model-based explanations and argue that upon closer examination they rather are 'model-induced explanations'. They take this to undermine the consensus view of what makes model-based explanations special or interesting, and they offer a new view on what makes explanations (generated) with models special.

In 'Scientific Explanation and Trade-offs between Explanatory Virtues', Alirio Rosales and Adam Morton take the plurality of scientific explanation as their starting point: 'explanation' refers to a wide range of activities with family resemblance between them. Rosales and Morton explore the limits of explanation. They argue that there are typically trade-offs in explanation, so that in strengthening one explanatory virtue one will usually weaken another. Scientific explanations are constrained by such trade-offs. This undermines the idea that explanations can be improved by maximizing different explanatory virtues simultaneously.

The diversity of legitimate scientific explanations does not necessarily imply that explanation is completely disunified: there may well be important similarities between various types of explanations. Hence an explanatory pluralist can also look for necessary conditions for explanations. In 'How to Reconcile a Unified Account of Explanation with Explanatory Diversity', Collin Rice and Yasha Rohwer argue that none of the features identified by existing accounts of explanation is necessary for all explanations. However, they argue that nevertheless a unified account can be developed by reconceiving of scientific explanation as a cluster concept. The main idea is that there are multiple subsets of features that are sufficient for providing an explanation, but no single feature is necessary for all explanations. Reconceiving of explanation as a cluster concept accounts for the diversity of kinds of explanations while preserving some degree of unity. 
Publisher's Note Springer Nature remains neutral with regard to jurisdictional claims in published maps and institutional affiliations.

Erik Weber is a professor in philosophy of science at Ghent University (Belgium) and director of the Ghent Centre for Logic and Philosophy of Science. Most of his research relates to general theories of causation and explanation, or to problems relating to causation and explanation in specific scientific disciplines (mathematics, psychology and the social sciences). He also works on the application of non-classical logics in the philosophy of science. His articles have appeared in journals such as Analysis, Economics \& Philosophy, Erkenntnis, Philosophia Mathematica, Philosophy of the Social Sciences, Studies in History and Philosophy of Science and Synthese.

Henk W. de Regt is professor of philosophy of natural sciences at the Institute for Science in Society, Radboud University, The Netherlands. He holds an MSc degree in foundations of physics (Utrecht University) and a PhD in philosophy (Vrije Universiteit Amsterdam). His current research focuses on scientific understanding and explanation, and on public understanding of science. His work has been published in journals such as Philosophy of Science, Synthese, Studies in History and Philosophy of Science, Journal for General Philosophy of Science, and Foundations of Science. His monograph Understanding Scientific Understanding (Oxford University Press, 2017) won the 2019 Lakatos Award.

Dingmar van Eck is postdoctoral researcher in philosophy of science at Ghent University, Belgium, and assistant professor in philosophy of science at the University of Amsterdam, the Netherlands. Most of his current research is on issues related to scientific explanation in the life sciences and engineering sciences, such as the modeling of mechanisms, the interplay between mechanistic and design explanations, and ontic and epistemic conceptions of scientific explanation. His articles have appeared in journals such as the British Journal for Philosophy of Science, European Journal for Philosophy of Science, Studies in History and Philosophy of Science, Philosophical Psychology and Erkenntnis. 